\title{
Phase II study of chidamide in combination with cisplatin in patients with metastatic triple-negative breast cancer
}

\author{
Yanchun Meng ${ }^{1,2 \#}$, Juan Jin ${ }^{1,2 \#}$, Chengcheng Gong ${ }^{1,2}$, Haitao Miao ${ }^{1,2}$, Zhonghua Tao ${ }^{1,2}$, Ting Li $^{1,2}, \mathrm{Jun} \mathrm{Cao}^{1,2}$, \\ Leiping Wang ${ }^{1,2}$, Biyun Wang ${ }^{1,2}$, Jian Zhang ${ }^{1,2}$, Xichun $\mathrm{Hu}^{1,2}$ \\ ${ }^{1}$ Department of Medical Oncology, Fudan University Shanghai Cancer Center, Shanghai, China; ${ }^{2}$ Department of Oncology, Shanghai Medical \\ College, Fudan University, Shanghai, China \\ Contributions: (I) Conception and design: J Zhang, X Hu; (II) Administrative support: L Wang, B Wang; (III) Provision of study materials or patients: \\ Y Meng, J Jin, L Wang, B Wang; (IV) Collection and assembly of data: C Gong, H Miao; (V) Data analysis and interpretation: Y Meng, J Jin, Z Tao, \\ T Li, J Cao; (VI) Manuscript writing: All authors; (VII) Final approval of manuscript: All authors. \\ \#These authors contributed equally to this work. \\ Correspondence to: Xichun Hu, MD, PhD; Jian Zhang, MD, PhD. Department of Medical Oncology, Fudan University Shanghai Cancer Center, No. \\ 255, Dong'An Road, Shanghai 200127, China. Email: huxichun2017@163.com; syner2000@163.com.
}

Background: Platinum-based regimens are the mainstay treatments for advanced triple-negative breast cancer (TNBC). Preclinical studies have shown that the histone deacetylase (HDAC) inhibitor chidamide induced antitumor effects in TNBC, and chidamide plus chemotherapy was shown to be tolerable in several malignancies. This study sought to investigate the efficacy and safety of a combination treatment of chidamide and cisplatin in metastatic TNBC patients.

Methods: In this phase II, single-arm study, women with metastatic TNBC were administered chidamide (20 mg twice weekly for 2 weeks on a 21 -day cycle) and cisplatin $\left(75 \mathrm{mg} / \mathrm{m}^{2}\right.$ on a 21 -day cycle). The primary endpoint was the objective response rate (ORR) by RECIST 1.1. The severity of adverse events was measured by the CTCAE 4.03 .

Results: Sixteen patients were enrolled in this study. Of these, 15 were available for evaluation. In these 15 patients, confirmed objective responses were seen in 4 patients [26.67\%, 95\% confidence interval (CI): $10.9 \%, 51.95 \%$ ]. The ORRs did not meet the predefined criteria (of a response by at least 5 of the 15 patients); thus, the study remained at stage I. The median progression-free survival (PFS) was 9.8 weeks; 4 patients had a PFS of $>25$ weeks. In relation to the treatment-related AEs $\geq$ grade $3,>2$ patients had neutropenia (33\%), thrombocytopenia (20\%), leucopenia (20\%), and vomiting (20\%).

Conclusions: The addition of chidamide did not improve the efficacy of cisplatin in the first-line treatment against advanced TNBC; thus, the phase II clinical trial did not progress any further. Our study appears to be the first to investigate the HDAC inhibitor in TNBC patients and showed disappointing results, which should inform future studies. Future research on cisplatin-based combination treatments for TNBC should consider selecting patients based on predictive biomarkers to increase the clinical benefits.

Keywords: Triple-negative breast cancer (TNBC); chidamide; cisplatin; combinational treatment

Submitted May 06, 2021. Accepted for publication Sep 09, 2021.

doi: 10.21037/apm-21-1139

View this article at: https://dx.doi.org/10.21037/apm-21-1139 


\section{Introduction}

Triple-negative breast cancer (TNBC) is defined as breast cancer that lacks the expression of estrogen receptor (ER), progesterone receptor (PR), and human epidermal growth factor receptor 2 (HER-2). It accounts for approximately $15 \%$ of all invasive breast cancers (1). An aggressive manner characterizes TNBC; patients are often at an advanced stage when diagnosed (1). As TNBC lacks hormone receptor (HR) and HER-2 expression, clinical benefits cannot be obtained from anti-hormone and anti-HER-2 therapies. Due to the high heterogeneity of TNBC, it is not easy to define appropriate targets for treatment. Thus, chemotherapy is the only option available in the therapeutic armamentarium (1). The 5 -year cancer-specific survival is $>85 \%$ in stage I TNBC patients and $>99 \%$ in stage I HR+/HER2- patients (2). The gap in overall survival (OS) between advanced TNBC patients and advanced breast cancer patients with other subtypes is huge. Metastatic TNBC patients have a median OS of about 1 year, while patients with other subtypes have a median OS of 5 years (2).

Platinum-based chemotherapy is the preferred therapeutic option for metastatic TNBC $(3,4)$. Platinum salts can bind to DNA to form DNA-platinum adducts, which produce double-strand breaks (DSBs). About $15 \%$ of TNBC patients harbor BRCA1/2 mutation, and at least $25 \%$ of $B R C A 1 / 2$ wild-type TNBC tumors exhibit "BRCAness", defined as molecular characteristics shared between sporadic tumors and carriers of BRCA1/2 mutations (5). The loss of BRCA1/2 function induces a deficiency in homologous recombination (HR), which is responsible for DSB repair. Thus, patients with BRCA1/2 deficient tumors are sensitive to platinum salt treatments. A recent meta-analysis showed that platinum regimens improved progress-free survival (PFS)/time to progression (TTP) in metastatic TNBC $(3,5,6)$. Unfortunately, not all patients benefit from platinum salts, and some develop drug resistance, leading to disease progression or recurrence. Research has shown that the overall response rate (ORR) of metastatic TNBC patients in platinum-based clinical trials is about $30 \%$, and the OS time is only about 12 months $(2,3,7)$. Notably, the treatment of advanced TNBC remains a critical clinical challenge, and the clinical efficacy of platinum slats in cancer treatment needs to be improved. Various studies have explored available adjunctive agents based on platinum-based regimens, including DNA-damage response-targeting cancer therapy, programmed death-1/ programmed death-ligand 1 (PD-1/PD-L1) inhibitors, and bevacizumab. However, very few studies have investigated the effects of combinational treatment strategies based on platinum-based regimens for TNBC patients, and no positive results for breast cancer patients have been demonstrated previously (8-11).

Histone deacetylases (HDACs), which catalyze the acetylation of histone proteins and contribute to aberrant gene expression in cancer cells, have been revealed to be dysregulated in a variety of malignant tumors, including breast cancer, which promotes the progression of cancer (12). Four different classes of HDACs (i.e., class I, II, III and IV) have been defined based on their homology. Several smallmolecule inhibitors targeting HDACs have been identified as effective treatment modalities against tumors (13). Chidamide was originally investigated as an oral HDAC inhibitor (14). It exerts potent antitumor activities by inhibiting the major class I (HDAC) subtypes, and the Chinese Food Drug Administration has approved it for the treatment of relapsed or refractory peripheral T-cell lymphoma (14). Chidamide in combination with antihormone therapy has shown significant efficacy in treating HR-positive advanced breast cancer in clinical practice $(15,16)$. Preclinical data indicate that chidamide inhibits the proliferation and migration of TNBC cells (17-19). The most common adverse events (AEs) related to chidamide are fatigue, thrombocytopenia, anorexia, and leucopenia (20). When combined with chemotherapy, hematologic toxicities, including neutropenia, thrombocytopenia, and leucopenia, are common. In a previous study of chidamide combined with paclitaxel and carboplatin in advanced non-small cell lung cancer patients, grade $3 / 4$ neutropenia in every cycle was observed in all patients (21).

Recent studies have shown that HDAC inhibitors, especially class I-HDAC inhibitors, induce significant synergistic effects with cisplatin against hematological and solid tumors, including in high-grade serous ovarian cancer, which shares similar molecular characteristics to TNBC (22-27). Further, histone hypoacetylation has been shown to condense the heterochromatin structure of DNA, which decreases the expression of tumor suppressor genes and blocks access to DNA-damaging agents like cisplatin. Thus, HDAC inhibitor treatment loosens the abnormally condensed heterochromatin structure of DNA, providing access to cisplatin (24). Based on this preclinical evidence, we conducted the first clinical trial on the efficacy and safety of a combinational chidamide and cisplatin treatment in advanced TNBC patients to further improve the clinical response of platinum agents and the prognosis of TNBC 
patients. We present the following article in accordance with the TREND reporting checklist (available at https:// dx.doi.org/10.21037/apm-21-1139).

\section{Methods}

\section{Patients}

To be eligible to participate in this study, patients had to meet the following criteria: (I) be a female aged 18 to 75 years with an Eastern Cooperative Oncology Group performance status of 0 or 1 , and a life expectancy of $\geq 3$ months; (II) have histologically confirmed recurrent and metastatic breast cancer, negative for ER and PR as confirmed by immunohistochemistry tests, and HER2 amplification as confirmed by fluorescence in situ hybridization; (III) have only undergone 1 previous line of chemotherapy without cisplatin or without the development of cisplatin resistance, which was defined as disease progress $<3$ months after the last cisplatin treatment; and (IV) have a measurable extracranial disease by RECIST 1.1; (V) and have acceptable hematological, hepatic, renal, and cardiac functions. Patients were excluded from the study if they met any of the following exclusion criteria: (I) had undergone previous treatment with HDAC inhibitors; (II) had symptomatic or uncontrolled central nervous system metastases; (III) had symptomatic serosal effusion or had received percutaneous drainage within the last 4 weeks; (IV) could not swallow, an intestinal obstruction, or gastrointestinal disorders that would affect the absorption of the study drug; ( V) had been administered any investigational agents within 30 days before study entry; (VI) had a history of another malignancy in the past 5 years other than cured basal cell carcinoma or in situ cervical carcinoma; (VII) had an uncontrolled infection, immunocompromised disease, or other severe concurrent illness; (VIII) had a history of the neurological or psychogenic disorder, such as epilepsy or dementia; (IX) were pregnant or had childbearing potential and were unwilling to use adequate contraception. All patients provided written informed consent before study entry.

\section{Treatment plan and study design}

This open-label, single-arm, 2-stage, phase II clinical trial was conducted at the Fudan University Shanghai Cancer Center in China according to the Declaration of Helsinki (as revised in 2013). The study was approved by Fudan
University Shanghai Cancer Center Institutional Review Board (No. 1910208-9), and informed consent was obtained from all patients. Patients received $30 \mathrm{mg}$ of a single-agent chidamide twice a week for 1 week in the run-in period. Patients received a combination treatment of $20 \mathrm{mg}$ of oral chidamide twice a week (biw) for the first 2 weeks of every 3 weeks, and $75 \mathrm{mg} / \mathrm{m}^{2}$ of cisplatin on day 1 every 3 weeks for $6-8$ cycles. Then, patients continued to take chidamide and $25 \mathrm{mg} / \mathrm{m}^{2}$ of cisplatin to maintain the response unless the patient had disease progression or unacceptable AEs. Patients gave a complete history, underwent laboratory assessments and physical examinations before study entry and every cycle, and underwent radiographic evaluations every 2 cycles to determine tumor response based on RECIST (version 1.1). Toxicities were graded using the Common Terminology Criteria for Adverse Events (version 4.03).

\section{Statistical analysis}

This phase II study used Simon's optimal 2-stage phase 2 design (28). P1 (projected ORR) was 0.50 , and $\mathrm{P} 0$ (previously reported ORR) was $0.30(\alpha=0.05$ and $\beta=0.80)$. It was decided that the results of this study would be considered negative if $\leq 5$ of the 15 patients had responses in the first stage of accrual. Otherwise, the study would move to the second stage of accrual, and another 31 patients would be added. If at least 19 of the 46 patients had a complete response (CR) or partial response (PR), the probability of the efficacy of treatment was $80 \%$. Allowing for a dropout rate of $15 \%$, it was determined that a sample size of 55 was needed.

The data were analyzed and processed using IBM SPSS Statistics software (version 25.0; IBM Corporation, Armonk, NY). No formal hypotheses were tested in this study, and all analyses were descriptive and exploratory. The primary endpoint was ORR, which was defined as CR and PR. The secondary endpoints included PFS and the clinical benefit rate $[\mathrm{CR}+\mathrm{PR}+$ stable disease (SD) for 24 weeks], and the evaluation of the side-effect profile of this combination. AEs were tabulated based on severity and frequency. $95 \%$ exact confidence intervals (CIs) were calculated for all response rates by normal approximation.

\section{Results}

\section{Patient characteristics}

Between January 2020 and September 2020, 16 patients 


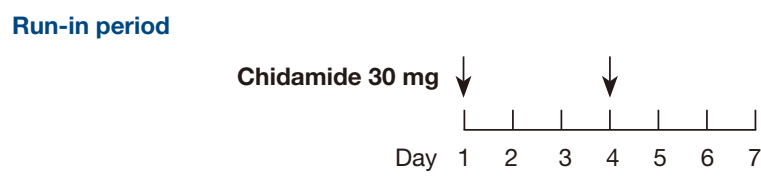

Combination treatment

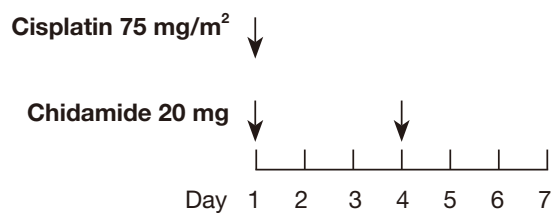

Maintenance treatment

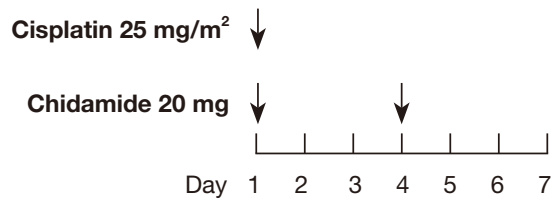

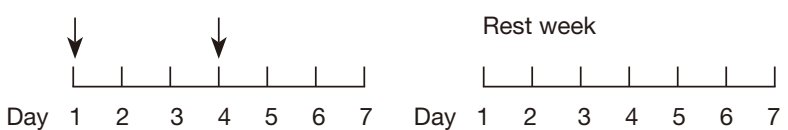

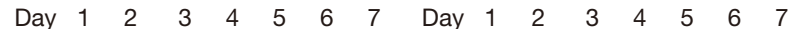

Figure 1 The treatment regimes.

with metastatic TNBC were enrolled in this study. The treatment regimens are depicted in Figure 1. Patient demographics and baseline characteristics are summarized in Table 1. Fifteen patients were evaluated for toxicity and efficacy. Due to the outbreak of coronavirus disease 2019, 1 of the 16 patients only received chidamide monotherapy for 5 weeks and died from acute coronary syndrome (ACS) before the combination treatment was initiated. 12 (75\%) patients had received no previous treatment for metastatic disease. Eleven (68.75\%) patients developed the metastatic disease within 1 year of radical mastectomy. Consistent with previously reported data (1), the most metastatic site was the lungs. Of 12 patients for whom BRCA1/2 mutation status was known, 2 (16.67\%) harbored a germline BRCA1 mutation; no BRCA2 mutation was found. The median number of treatment cycles was 2 (range, 1 to 10). 3 patients were still receiving treatment at the cut-off date (January 10,2021 ), with treatment durations of 221,213 , and 139 days, respectively.

\section{Efficacy}

Among the 15 patients available for evaluation (Table 2), no CR was observed. Four patients reached PR, and among those, 2 patients were still being treated at the cut-off date and had been receiving treatment for 31 weeks and 19 weeks, respectively. The patient who had shown a PR for 31 weeks had multiple lung metastases. Her lung metastases shrunk after 2 cycles and were not obvious after eight cycles. Another 2 patients with PR, receiving treatment for 6 weeks and 19 weeks, respectively, both had a germline BRCA1 mutation. The ORR (CR + PR) was 4 in 15 patients (26.67\%, 95\% CI: $10.9 \%, 51.95 \%) .3$ patients had SD that received treatment for 19,26 , and 27 weeks, respectively. The clinical benefit rate (CR + PR + SD > 24 weeks) was 6 in 15 patients (40\%, 95\% CI: 19.82\%, 64.25\%). The median PFS was 9.8 weeks. The results did not meet the predefined efficacy criteria for further evaluation.

\section{Toxicity}

Fifteen patients who completed at least 1 cycle of combinational treatment were evaluated for treatmentrelated AEs (Table 3). One patient enrolled on January 25, 2021 only received chidamide monotherapy $(30 \mathrm{mg}$ biw) for 5 weeks when coronavirus disease 2019 began to emerge in China. On March 9, 2021, after treatment of chidamide monotherapy, this patient, who had bone and liver metastasis, developed grade 3 increased aspartate aminotransferase. On March 13, 2021, this patient was diagnosed with ACS. On March 26, 2021, this patient died from respiratory failure and ACS. The other AEs of these patients were mild and included thrombocytopenia, constipation, and hypokalemia. No patients discontinued 
Table 1 Patient characteristics

\begin{tabular}{lc}
\hline Characteristic & No. of patients $(n=16)$ \\
\hline Age (years), mean (range) & $47(25$ to 65$)$ \\
ECOG performance status & \\
0 & 2 \\
1 & 14
\end{tabular}

Time between breast surgery and metastatic disease

$\begin{array}{ll}\text { Metastatic } & 2 \\ <1 \text { year } & 11 \\ \geq 1 \text { year } & 3\end{array}$

Number of metastatic organ sites

$\begin{array}{lc}1 & 4 \\ 23 & 4 \\ \text { Metastatic sites } & 10 \\ \text { Lung } & 8 \\ \text { Liver } & 2 \\ \text { Pleura } & 4 \\ \text { Bone } & 9 \\ \text { Lymph nodes } & \\ \text { Neoadjuvant or adjuvant chemotherapy } & 11 \\ \text { Anthracycline } & 10 \\ \text { Taxane } & 1 \\ \text { Platinum salt } & 2 \\ \text { Capecitabine } & \end{array}$

Number of previous treatments

0

1

BRCA1/2 mutation status

Germline BRCA1 mutation 2

BRCA1/2 wild-type 10

ECOG, Eastern Cooperative Oncology Group Performance; BRCA1/2, breast cancer gene 1 and 2 .

treatment due to AEs. Nine (60\%) patients experienced grade $3 \mathrm{AEs}$, and 3 (20\%) patients experienced grade $4 \mathrm{AEs,}$ including neutropenia, thrombocytopenia, and hypokalemia. Three patients had dose reductions of both cisplatin and chidamide due to vomiting $(\mathrm{n}=2)$ and thrombocytopenia
Table 2 Response summary

\begin{tabular}{lc}
\hline Best response & Number (\%) \\
\hline Partial response & $4(26.67)$ \\
Stable disease & $3(20.00)$ \\
Progressive disease & $8(53.33)$ \\
\hline
\end{tabular}

$(\mathrm{n}=1)$, and 1 patient had a chidamide dose reduction only for hypokalemia $(\mathrm{n}=1)$.

The most common hematological toxicities were anemia (87\%) and leucopenia (73\%). 7 (46.67\%) patients experienced grade $3 / 4$ myelosuppression. Two (13\%) patients experienced grade IV neutropenia that was resolved by the administration of colony-stimulating factor and did not affect treatment. No patient had febrile neutropenia during the study. One patient had grade IV thrombocytopenia, which led to the hospitalization and a dose reduction of cisplatin and chidamide. The most common non-hematological toxicities were fatigue (53\%) and gastrointestinal toxicities, including nausea (93\%), anorexia (53\%), vomiting (53\%), and constipation (53\%), most of which were mild. As all patients took aprepitant to prevent nausea and vomiting, most patients only experienced grade I nausea (93\%) or vomiting (33\%); however, 3 (20\%) patients experienced grade III vomiting, and 1 of these 3 patients required hospitalization and a dose reduction of cisplatin and chidamide. Laboratory abnormalities were observed, most of which were associated with electrolyte disturbance, including hyponatremia (49\%) and hypokalemia (40\%). 1 patient experienced grade IV hypokalemia and 2 patients experienced grade III increased alanine aminotransferase. Other laboratory abnormalities included hyperglycemia (40\%) and hypoalbuminemia (40\%).

\section{Discussion}

Chemotherapy is integral to the treatment of advanced TNBC (1). Single-agent platinum-salt chemotherapy has been shown to induce an ORR of $31 \%$ (2). In a randomized, multicenter, phase 3, CBCSG006 trial, cisplatin plus gemcitabine was shown to be superior to paclitaxel plus gemcitabine as a first-line therapy for metastatic TNBC patients (PFS: 7.73 vs. 6.47 months, respectively) (4). Platinum salts have become the cornerstone of treatment for advanced TNBC; however, as a plateau has been reached in traditional chemotherapy, novel molecularly targeted agents combined with platinum salts need to 
Table 3 Incidence of adverse events

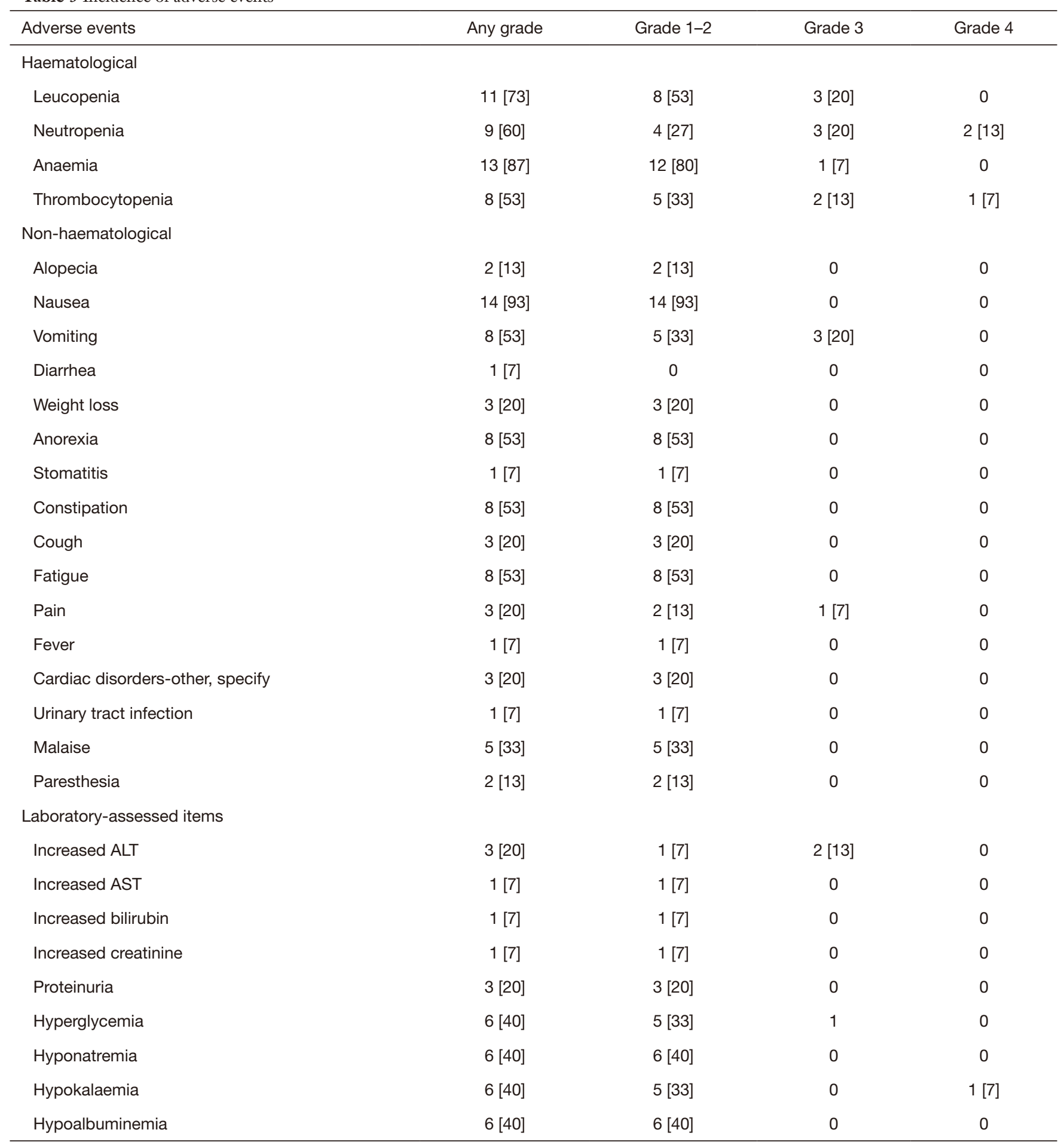

Data are $\mathrm{n}[\%]$ from the safety population. ALT, alanine aminotransferase; AST, aspartate aminotransferase. 
be evaluated. Overexpressed HDAC1/HDAC2 and a specific pattern of expression of HDAC1, 2, 3, and 6 have been observed in malignant breast neoplasms (29). Several clinical trials are being conducted to test HDAC inhibitor monotherapy alone or combined with standard cytotoxic agents and novel targeted agents in patients with TNBC $(30,31)$. In this prospective, single-center, phase II trial, we first investigated the efficacy and safety of an HDAC inhibitor plus cisplatin in TNBC patients. To our knowledge, this was the first prospective trial conducted to investigate the efficacy of an HDAC inhibitor in treating patients with TNBC. We chose to combine chidamide with cisplatin for a number of reasons. First, chidamide combined with DNA-damaging agents, including cisplatin, had shown activity against in several studies $(19,22,24,25)$. Second, preclinical data had shown that chidamide inhibited the proliferation and migration of TNBC cells (19). Third, the molecular mechanisms of the chidamide/cisplatin against tumors provided a rationale for their synergistic effect (24). However, TNBC is of high heterogeneity and aggressiveness, rendering clinical investigations much more difficult. Our results revealed the disappointing efficacy of HADC inhibitors in TNBC.

In the current study, the pre-specified efficacy threshold (a response by 5 of the 15 patients ) was not met; no patients achieved CR, and only 4 of the 15 (26.67\%) patients achieved PR. The ORR of platinum-based chemotherapy was previously reported to be more than $30 \%$ in metastatic patients with TNBC $(7,32)$. The median PFS in the current trial was 9.8 weeks, which was much lower than that previously reported (4). Most patients in the current study received the combination treatment as first-line chemotherapy and had no previous cisplatin exposure. Our study's lower ORR and PFS could be attributed to the different combinational treatment strategies and the small study size. Additionally, $30 \mathrm{mg}$ of chidamide biw is recommended as the dose to be combined with anti-hormone therapy for HR-positive breast cancer or chemotherapy for $\mathrm{T}$ lymphoblastic lymphoma/leukemia $(15,33)$. In our study, to control the treatment-related AEs, we chose to combine $20 \mathrm{mg}$ of chidamide biw with cisplatin; however, this amount might have been insufficient to induce any pronounced activity against tumors. Given the good tolerability of chidamide plus chemotherapy in breast cancer, a higher dose of chidamide could be considered in future research. Sufficient evidence was not found to continue to the next stage in this trial, as only 4 patients achieved PR and 3 patients achieved SD .

According to previous studies, the most common AEs associated with platinum regimens are nausea, vomiting, anorexia, anemia, and thrombocytopenia, and the most frequently reported AEs associated with chidamide are fatigue thrombocytopenia, neutropenia, nausea, anorexia, vomiting, and diarrhea $(6,16)$. The most frequent AEs reported in the current study were gastrointestinal events, including nausea (93\%) and vomiting (53\%), anorexia (53\%), constipation (53\%), hematological events, including leucopenia (73\%), neutropenia (60\%), anemia (87\%), and thrombocytopenia $(53 \%)$, and fatigue (60\%). The grade of gastrointestinal and hematological AEs in the current study was apparently higher than those of chidamide monotherapy in peripheral T-cell lymphoma patients (34). This might be due to the overlapping toxicities of each drug in the combination treatment. Most AEs were mild to moderate, and dose discontinuation was not required. Grade 3/4 myelosuppressive events were observed in our clinical trial; however, consistent with the findings of previous studies $(35,36)$, these events were transient and reversible. Considering the common gastrointestinal and myelosuppressive events related to the current regimens, further clinical trials on combinational treatments of HADC inhibitors and platinum salts should seek to monitor the symptoms and establish appropriate management strategies.

This trial had several limitations, including its small sample size, non-randomized character, and the un-selection of the enrolled patients. Further biomarker analysis of our CBCSG006 trial investigating cisplatin-based chemotherapy in TNBC demonstrated that HR deficiency status was significantly associated with higher ORR and longer PFS in a cisplatin plus gemcitabine treatment than a paclitaxel plus gemcitabine treatment in metastatic TNBC (37). In the current study, the 2 patients with germline BRCA1 mutation achieved PR, which provides further evidence of the predictive role of the $B R C A 1 / 2$ mutation in cisplatin treatment. Thus, selecting appropriate patients for the clinical study of TNBC is critical.

Together, the combination of chidamide and cisplatin does not appear to have superior efficacy to standard cisplatin-based chemotherapy in treating advanced TNBC. Our study's limited ORR and PFS reflected the treatmentrefractory nature of such tumors and the urgent need for more effective therapies. Several studies investigating the efficacy and safety of adding novel targeted agents, 
including WEE1 inhibitors and PD-L1 inhibitors, to platinum-based chemotherapy in TNBC have been conducted (38-40). Chidamide has been observed to enhance immune cell-mediated antitumor activity (41). Several ongoing clinical trials investigate the efficacy and safety of combination treatments of immune checkpoint inhibitors and other HDAC inhibitors in TNBC (30). Thus, combining chidamide with immune checkpoint inhibitors may represent a promising approach for further clinical investigations in TNBC. It will be interesting to see whether novel combination strategies improve the outcome of platinum agents in TNBC patients.

\section{Acknowledgments}

Funding: This study was supported by National Natural Science Foundation of China (grant No. 82072915), the Shanghai Municipal Science and Technology Commission Guidance Project, China (contract No. 18411967800), research grant from Shanghai Hospital Development Center (grant No. SHDC12018X03), and CSCO-ROCHE Cancer Research Fund 2019 (grant No. Y-2019Roche-171).

\section{Footnote}

Reporting Checklist: The authors have completed the TREND reporting checklist. Available at https://dx.doi. org/10.21037/apm-21-1139

Data Sharing Statement: Available at https://dx.doi. org/10.21037/apm-21-1139

Conflicts of Interest: All authors have completed the ICMJE uniform disclosure form (available at https://dx.doi. org/10.21037/apm-21-1139). JZ serves as an unpaid editorial board member of Annals of Palliative Medicine from January 2020 to December 2021. The authors have no other conflicts of interest to declare.

Ethical Statement: The authors are accountable for all aspects of the work in ensuring that questions related to the accuracy or integrity of any part of the work are appropriately investigated and resolved. This open-label study was conducted according to the Declaration of Helsinki (as revised in 2013). The study was approved by Fudan University Shanghai Cancer Center Institutional Review Board (1910208-9), and informed consent was obtained from all patients.
Open Access Statement: This is an Open Access article distributed in accordance with the Creative Commons Attribution-NonCommercial-NoDerivs 4.0 International License (CC BY-NC-ND 4.0), which permits the noncommercial replication and distribution of the article with the strict proviso that no changes or edits are made and the original work is properly cited (including links to both the formal publication through the relevant DOI and the license). See: https://creativecommons.org/licenses/by-nc-nd/4.0/.

\section{References}

1. Foulkes WD, Smith IE, Reis-Filho JS. Triple-negative breast cancer. N Engl J Med 2010;363:1938-48.

2. Waks AG, Winer EP. Breast Cancer Treatment: A Review. JAMA 2019;321:288-300.

3. Gerratana L, Fanotto V, Pelizzari G, et al. Do platinum salts fit all triple negative breast cancers? Cancer Treat Rev 2016;48:34-41.

4. Hu XC, Zhang J, Xu BH, et al. Cisplatin plus gemcitabine versus paclitaxel plus gemcitabine as first-line therapy for metastatic triple-negative breast cancer (CBCSG006): a randomised, open-label, multicentre, phase 3 trial. Lancet Oncol 2015;16:436-46.

5. Jin J, Zhang W, Ji W, et al. Predictive biomarkers for triple negative breast cancer treated with platinum-based chemotherapy. Cancer Biol Ther 2017;18:369-78.

6. Egger SJ, Chan MMK, Luo Q, et al. Platinum-containing regimens for triple-negative metastatic breast cancer. Cochrane Database Syst Rev 2020;10:CD013750.

7. Tutt A, Tovey H, Cheang MCU, et al. Carboplatin in BRCA1/2-mutated and triple-negative breast cancer BRCAness subgroups: the TNT Trial. Nat Med 2018;24:628-37.

8. Basourakos SP, Li L, Aparicio AM, et al. Combination Platinum-based and DNA Damage Response-targeting Cancer Therapy: Evolution and Future Directions. Curr Med Chem 2017;24:1586-606.

9. Gadgeel S, Rodríguez-Abreu D, Speranza G, et al. Updated Analysis From KEYNOTE-189: Pembrolizumab or Placebo Plus Pemetrexed and Platinum for Previously Untreated Metastatic Nonsquamous Non-Small-Cell Lung Cancer. J Clin Oncol 2020;38:1505-17.

10. Xue Y, Gao S, Gou J, et al. Platinum-based chemotherapy in combination with PD-1/PD-L1 inhibitors: preclinical and clinical studies and mechanism of action. Expert Opin Drug Deliv 2021;18:187-203.

11. Pfisterer J, Shannon CM, Baumann K, et al. Bevacizumab 
and platinum-based combinations for recurrent ovarian cancer: a randomised, open-label, phase 3 trial. Lancet Oncol 2020;21:699-709.

12. Riaz SK, Saeed M, Malik M. Clinical and Therapeutic Implications of Histone Acetylation in Breast Cancer. West Indian Med J 2015;65:337-44.

13. Ediriweera MK, Tennekoon KH, Samarakoon SR. Emerging role of histone deacetylase inhibitors as antibreast-cancer agents. Drug Discov Today 2019;24:685-702.

14. Ye J, Zha J, Shi Y, et al. Co-inhibition of HDAC and MLL-menin interaction targets MLL-rearranged acute myeloid leukemia cells via disruption of DNA damage checkpoint and DNA repair. Clin Epigenetics 2019;11:137.

15. Jiang Z, Li W, Hu X, et al. Tucidinostat plus exemestane for postmenopausal patients with advanced, hormone receptor-positive breast cancer (ACE): a randomised, double-blind, placebo-controlled, phase 3 trial. Lancet Oncol 2019;20:806-15.

16. Zhang Q, Wang T, Geng C, et al. Exploratory clinical study of chidamide, an oral subtype-selective histone deacetylase inhibitor, in combination with exemestane in hormone receptor-positive advanced breast cancer. Chin J Cancer Res 2018;30:605-12.

17. Bai X, Jiang H, Han G, et al. Chidamide suppresses the glycolysis of triple negative breast cancer cells partially by targeting the miR-33a-5p-LDHA axis. Mol Med Rep 2019;20:1857-65.

18. Zhou W, Han H, Xu J, et al. Autophagic Vacuole Secretion Triggered by Chidamide Participates in TRAIL Apoptosis Effect in Breast Cancer Cells. Curr Pharm Des 2021;27:2366-80.

19. Bissonnette RP, Rolland A, Goodenow B, et al. The activity of the HDAC inhibitor HBI-8000 (Chidamide) combined with eribulin on 4T1 spontaneous metastasis development. J Clin Oncol 2017;35:e14055.

20. Dong M, Ning ZQ, Xing PY, et al. Phase I study of chidamide (CS055/HBI-8000), a new histone deacetylase inhibitor, in patients with advanced solid tumors and lymphomas. Cancer Chemother Pharmacol 2012;69:1413-22.

21. Hu X, Wang L, Lin L, et al. A phase I trial of an oral subtype-selective histone deacetylase inhibitor, chidamide, in combination with paclitaxel and carboplatin in patients with advanced non-small cell lung cancer. Chin J Cancer Res 2016;28:444-51.

22. Bandolik JJ, Hamacher A, Schrenk C, et al. Class I-Histone Deacetylase (HDAC) Inhibition is Superior to pan-HDAC Inhibition in Modulating Cisplatin Potency in High
Grade Serous Ovarian Cancer Cell Lines. Int J Mol Sci 2019;20:3052.

23. Krieger V, Hamacher A, Cao F, et al. Synthesis of Peptoid-Based Class I-Selective Histone Deacetylase Inhibitors with Chemosensitizing Properties. J Med Chem 2019;62:11260-79.

24. Pchejetski D, Alfraidi A, Sacco K, et al. Histone deacetylases as new therapy targets for platinum-resistant epithelial ovarian cancer. J Cancer Res Clin Oncol 2016;142:1659-71.

25. To KKW, Fu LW. CUDC-907, a dual HDAC and PI3K inhibitor, reverses platinum drug resistance. Invest New Drugs 2018;36:10-9.

26. Zhou Y, Pan DS, Shan S, et al. Non-toxic dose chidamide synergistically enhances platinum-induced DNA damage responses and apoptosis in Non-Small-Cell lung cancer cells. Biomed Pharmacother 2014;68:483-91.

27. Zhang Y, Liu J, Raj-Kumar PK, et al. Development and validation of prognostic gene signature for basal-like breast cancer and high-grade serous ovarian cancer. Breast Cancer Res Treat 2020;184:689-98.

28. Simon's two-stage design. Available online: http:// cancer.unc.edu/biostatistics/program/ivanova/ Simons'TwoStageDesign.aspx. Accessed Jan 32020.

29. Zwergel C, Stazi G, Valente S, et al. Histone deacetylase inhibitors: updated studies in various epigenetic-related diseases. J Clin Epigenetics 2016;2:1. doi: 10.21767/24721158.100015

30. Trapani D, Esposito A, Criscitiello C, et al. Entinostat for the treatment of breast cancer. Expert Opin Investig Drugs 2017;26:965-71.

31. Huang Y, Nayak S, Jankowitz R, et al. Epigenetics in breast cancer: what's new? Breast Cancer Res 2011;13:225.

32. Fan $\mathrm{Y}, \mathrm{Xu} \mathrm{BH}$, Yuan $\mathrm{P}$, et al. Docetaxel-cisplatin might be superior to docetaxel-capecitabine in the first-line treatment of metastatic triple-negative breast cancer. Ann Oncol 2013;24:1219-25.

33. Guan W, Jing Y, Dou L, et al. Chidamide in combination with chemotherapy in refractory and relapsed $\mathrm{T}$ lymphoblastic lymphoma/leukemia. Leuk Lymphoma 2020;61:855-61.

34. Shi $\mathrm{Y}$, Dong M, Hong $\mathrm{X}$, et al. Results from a multicenter, open-label, pivotal phase II study of chidamide in relapsed or refractory peripheral T-cell lymphoma. Ann Oncol 2015;26:1766-71.

35. Subramanian S, Bates SE, Wright JJ, et al. Clinical Toxicities of Histone Deacetylase Inhibitors. Pharmaceuticals (Basel) 2010;3:2751-67. 
36. Barabas K, Milner R, Lurie D, et al. Cisplatin: a review of toxicities and therapeutic applications. Vet Comp Oncol 2008;6:1-18.

37. Zhang J, Lin Y, Sun XJ, et al. Biomarker assessment of the CBCSG006 trial: a randomized phase III trial of cisplatin plus gemcitabine compared with paclitaxel plus gemcitabine as first-line therapy for patients with metastatic triple-negative breast cancer. Ann Oncol 2018;29:1741-7.

38. Fremd C, Jaeger D, Schneeweiss A. Targeted and immunobiology driven treatment strategies for triple-negative breast cancer: current knowledge and future perspectives. Expert Rev Anticancer Ther 2019;19:29-42.

39. Keenan TE, Li T, Vallius T, et al. Clinical Efficacy and Molecular Response Correlates of the WEE1 Inhibitor

Cite this article as: Meng Y, Jin J, Gong C, Miao H, Tao Z, Li T, Cao J, Wang L, Wang B, Zhang J, Hu X. Phase II study of chidamide in combination with cisplatin in patients with metastatic triple-negative breast cancer. Ann Palliat Med 2021;10(11):11255-11264. doi: 10.21037/apm-21-1139
Adavosertib Combined with Cisplatin in Patients with Metastatic Triple-Negative Breast Cancer. Clin Cancer Res 2021;27:983-91.

40. Cortés J, André F, Gonçalves A, et al. IMpassion132 Phase III trial: atezolizumab and chemotherapy in early relapsing metastatic triple-negative breast cancer. Future Oncol 2019;15:1951-61.

41. Ning ZQ, Li ZB, Newman MJ, et al. Chidamide (CS055/ HBI-8000): a new histone deacetylase inhibitor of the benzamide class with antitumor activity and the ability to enhance immune cell-mediated tumor cell cytotoxicity. Cancer Chemother Pharmacol 2012;69:901-9.

(English Language Editors: L. Huleatt and J. Chapnick) 